\title{
Occipital cortex dysgenesis with white matter changes due to mutations in Laminin $\alpha 2$
}

\author{
Uluç Yiş ${ }^{1}$, Vishal Dixit ${ }^{5,6,7}$, Sedat Işıkay ${ }^{2}$, Mert Karakaya5,6,7, Figen Baydan ${ }^{3}$, \\ Gülden Diniz ${ }^{4}$, İpek Polat ${ }^{1}$, Semra Hız-Kurul ${ }^{1}$, Sebahattin Çırak ${ }^{5,6,7}$ \\ Division of Child Neurology, Department of Pediatrics, ${ }^{1}$ Dokuz Eylül University School of Medicine, İzmir, ${ }^{2}$ Sütçï İmam \\ University, School of Medicine, Kahramanmaraş and ${ }^{3,4}$ Tepecik Training and Research Hospital, İzmir, ${ }^{4}$ Neuromuscular \\ Disease Center, ${ }^{5}$ Institut für Humangenetik am Universitätsklinikum, Köln, ${ }^{6}$ Department of Pediatrics, University Hospital \\ Cologne, Cologne, ${ }^{7}$ Centre for Molecular Medicine, University Hospital Cologne, Cologne, Germany. \\ E-mail: ipekkalafatcilar@gmail.com
}

Receivd: 10th October 2016, Accepted: 7th November 2016

SUMMARY: Yiş U, Dixit V, Işıkay S, Karakaya M, Baydan F, Diniz G, Polat I, Hız-Kurul S, Çırak S. Occipital cortex dysgenesis with white matter changes due to mutations in Laminin a2. Turk J Pediatr 2017; 59: 338-341.

Laminin $\alpha 2$ related congenital muscular dystrophy is one of the most common congenital muscular dystrophies of childhood with or without clinical evidence of central nervous system involvement. It may be associated with significant white matter abnormalities resembling leukodystrophies. In this study, we elaborated on two cases with laminin $\alpha 2$ related congenital muscular dystrophy who had occipital cortex dysgenesis in addition to characteristic white matter abnormalities. Although laminin $\alpha 2$ related congenital muscular dystrophy with white matter abnormalities is known, the association with occipital cortex dysplasia has been not well recognized by clinical colleagues.

Key words: laminin $\alpha 2$, congenital muscular dystrophy, occipital cortex, dysgenesis.

The clinical manifestations of LAMA2-related muscular dystrophy (LAMA2 MD) range from severe, early-onset congenital muscular dystrophy (CMD) - referred to as early-onset LAMA2related muscular dystrophy (LAMA2 MD) to mild, later childhood-onset limb-girdle type muscular dystrophy as late-onset $L A M A 2$-related muscular dystrophy (LAMA2 MD) ${ }^{1}$. LAMA2 Laminin $\alpha 2$ related $C M D$ is caused by the mutations in the LAMA2 gene (OMIM 607855) located on chromosome 6q22-23 coding the $\alpha 2$ chain of laminin-211, also known as merosin, a heterotrimeric extracellular matrix protein ${ }^{2}$. It is one of the most common forms of CMD. Patients present with hypotonia, weakness of the extremities and contractures in the distal extremities. Patients with complete deficiency do not achieve independent ambulation but patients with a partial deficiency present with milder phenotypes ${ }^{1,2}$. Most of the patients have abnormal brain magnetic resonance imaging (MRI), which consists of high signal on T2 weighted and FLAIR images ${ }^{1,2}$. Malformations of cortical development mainly involving the occipital cortex have been not well appreciated in clinical practice to be associated with laminin $\alpha 2$ related congenital muscular dystrophy ${ }^{3-9}$.

\section{Case Reports}

Case 1

A four-month-old boy was admitted for the investigation of delayed motor milestones. Birth history was unremarkable. Parents were second-degree consanguineous and he had a healthy four-year-old sister. He was not able to hold his head. He was hypotonic and deep tendon reflexes could not be obtained. Serum creatinine kinase was elevated with 4267 IU/L. Congenital muscular dystrophy was suggested and the patient was referred for ophthalmologic examination and brain magnetic resonance imaging (MRI) scans. Ophthalmologic examination was normal but brain MRI revealed occipital cortical dysgenesis with a subcortical band heterotopia (Fig. 1a). There was also white matter hyperintensities in the bioccipital white matter. Supportive care in the form of physiotherapy was initiated. Informed consent was received from the 
family for detailed genetic analysis. We performed whole exome sequencing (WES) from peripheral blood DNA of the patient after Nimblegen enrichment (SeqCap EZ Human Exome Library v2.00) on the Illumina HiSeq 2000 platform with 2x100bp according to manufacturer instructions. The Coverage was 75 fold i.e., 10x coverage for $97 \%$ of target sequences and 30x coverage for $87.6 \%$ of target sequences. The Cologne Center for Genomics VARBANK pipeline v.2.12 (https:// varbank.ccg.uni-koeln.de/) was used for data analysis for rare autosomal recessive disease in a consanguineous family. We employed highly stringent criteria according to expected inheritance pattern, quality, frequency $(<0.1 \%)$ and pathogenicity. Data filtration revealed an autosomal recessive homozygous missense mutation p.R2578* (c.7732C > T) in the LAMA2 gene. The parents were heterozygous for the mutation. Finally, family was offered genetic counseling.

\section{Case 2}

A six month-old-boy was admitted for the investigation of generalized hypotonia and pes equinovarus deformity. He was the first child of non-consanguineous parents. The birth history was unremarkable except for foot deformities. On neurologic examination, he was able to hold his head for a few seconds but was not able to sit. Deep tendon reflexes could not be obtained. Serum creatine kinase value was 2529 IU/L. A muscle biopsy was obtained during corrective surgery for pes equinovarus deformity. Muscle biopsy of the patient revealed total deficiency of laminin $\alpha 2$ with dystrophic changes (Fig. 2). Brain MRI of the patient revealed occipital cortical dysgenesis, subcortical band heterotopia and bioccipital white matter hyperintensities (Fig. 1b). Informed consent was received from the family for detailed genetic analysis. Conventional diagnostic Sanger sequencing of the coding exons and flanking introns using DNA isolated from peripheral blood of the patient and both parents was performed.We found compound heterozygous mutations p.C1125Mfs*4 (c.3371 3372insA)/p.R2319* (c.6955C $>\mathrm{T}$ ) in the LAMA2. The mother (p.C1125Mfs*4 (c.3371_3372insA) and father

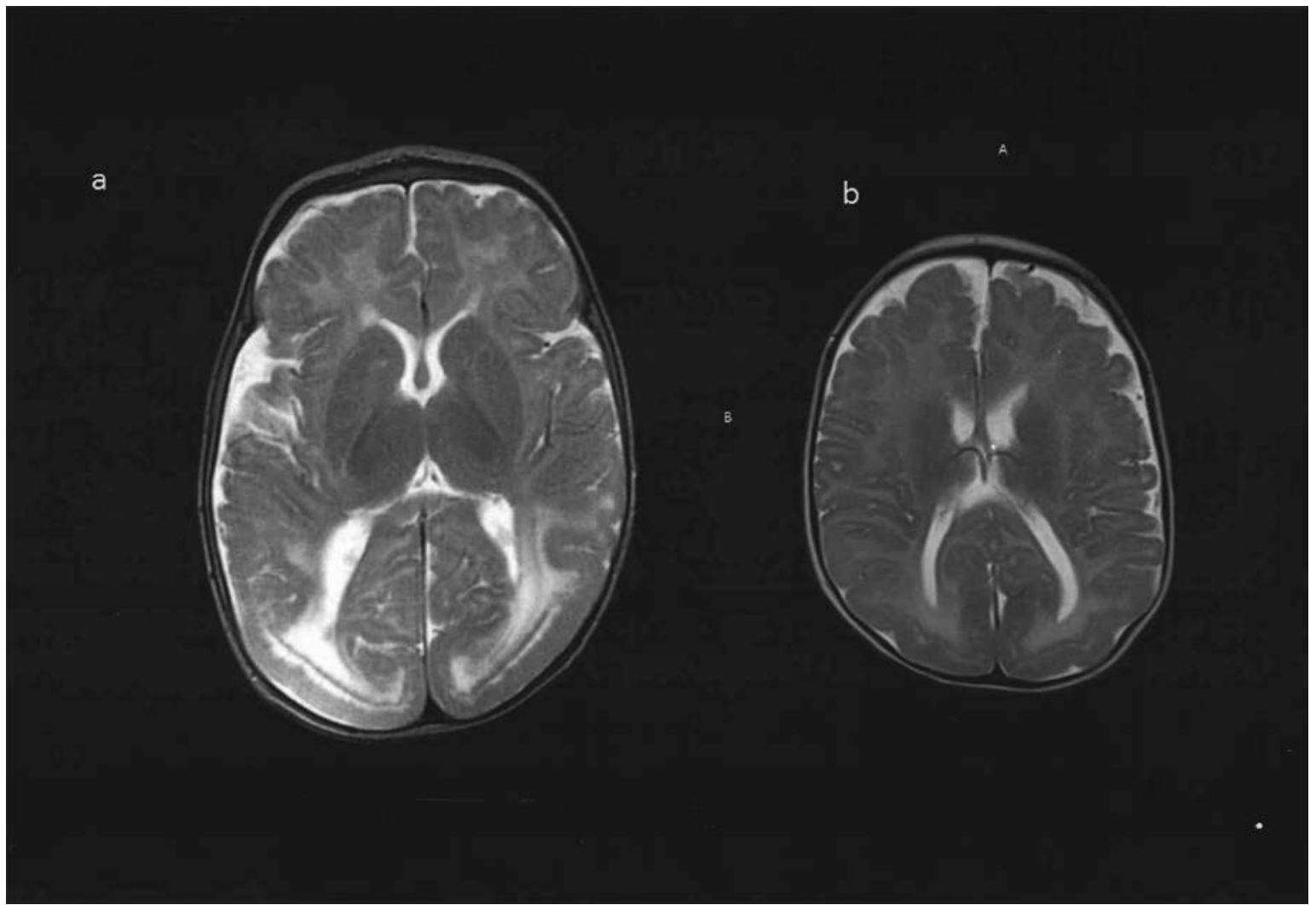

Fig. 1. Brain magnetic resonance imaging of the first (1a) and second (1b) patient revealed occipital cortical dysgenesis, subcortical band heterotopia and bioccipitial white matter hyperintensities 


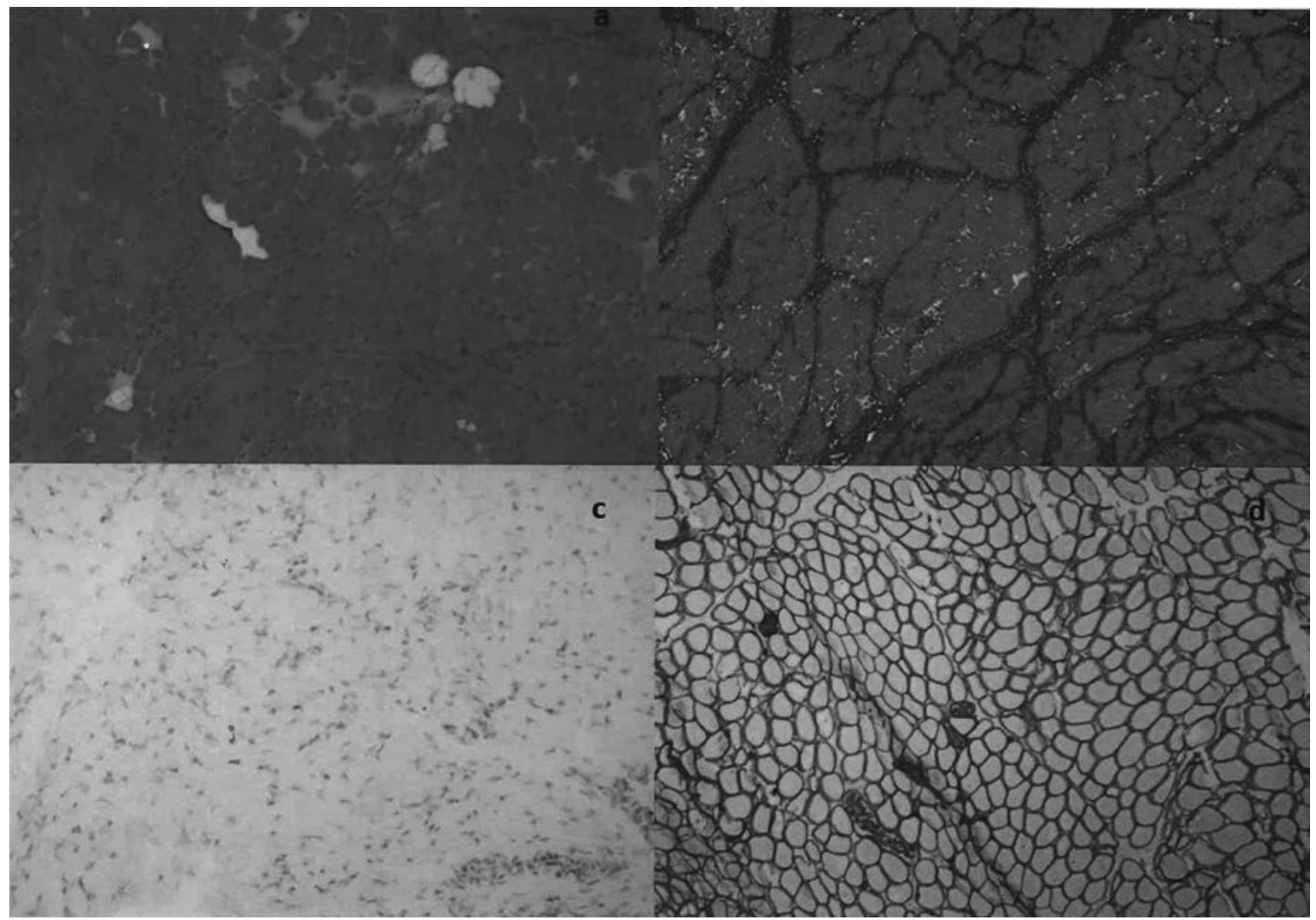

Fig. 2. Muscle biopsy of the second patient. Dystrophic presentation with increased connective tissue and fat, altered fasicles with small round muscle fibers of variable size (hematoxylin and eosin staining 200x) (2a); increased interstitial fibrous tissue (Masson's trichrome staining 40x) (2b); diffuse absence of merosin (DAB 200x) (2c); merosin positive control patient (DAB 100x)

(p.R2319* (c.6955C>T)) were heterozygous carriers for these mutations. Supportive care in the form of physiotherapy was initiated. Patient permission forms for publishment of cases were signed up by their parents

\section{Discussion}

The CMDs are a group of genetically and clinically heterogeneous hereditary myopathies possessing preferentially autosomal recessive inheritance, which are characterized by congenital hypotonia, delayed motor development and early onset of progressive muscle weakness associated with dystrophic pattern on muscle biopsy $^{1-3}$ Two types of CMD characterized by brain involvement are laminin $\alpha 2$ related congenital muscular dystrophy and alphadystroglycanopathies. Pachygyric/lissencephalic cortex with white matter abnormalities and prominent infratentorial involvement including cerebellar atrophy, cerebellar cysts, hypoplastic pons and thick tectum are main brain MRI findings in alpha dystroglycanopathies ${ }^{4}$. Brain involvement in laminin $\alpha 2$ related CMD is characterized by diffuse and symmetrical abnormal signal intensity in the supratentorial white matter. Abnormal signal intensity involves white matter hypointensity on T1-weighted images and hyperintensity on T2 weighted images. Frontal, temporal and parietal lobes are more severely affected than the occipital lobes. The corpus callosum has been spared in both cases. It is suggested that leakage of plasma components as a result of abnormal brain barrier caused by laminin $\alpha 2$ deficiency is responsible for white matter changes. Cerebellar and pons hypoplasia have also been described in some cases ${ }^{3}$.

Migrational disorders associated with white matter involvement have been rarely reported in laminin $\alpha 2$ related congenital muscular dystrophy. These disorders mainly include agyria/pachygyria and polymicrogyria of occipital cortex ${ }^{5,7}$. Leite et $\mathrm{al}^{5}$. described only 
one case of bioccipitial pachygyria in MRI series of 25 cases with laminin $\alpha 2$ related congenital muscular dystrophy. Tsao et al. ${ }^{7}$ reported two infants with complete laminin $\alpha 2$ deficiency who have not only abnormal cerebral white-matter lesions, but also bioccipital polymicrogyria. Geranmayeh et al. ${ }^{6}$ reported 51 cases with merosin deficient congenital muscular dystrophy. Thirty-four of these patients had brain magnetic resonance imaging. $3 / 34$ cases had normal scans, $18 / 34$ had typical white matter changes in periventricular and subcortical white matter and 13/31 had cortical abnormalities mainly including pachygyria and agyria in occipital lobes. Cortical malformations were both present in patients with total and partial merosin deficiency. Some patients with laminin $\alpha 2$ deficiency and focal cortical dysplasia in the posterior areas of brain may also have drug resistant occipital epilepsy ${ }^{7-9}$. These patients may have partial seizures characterized by clusters of epileptic spasms, which may be missed or misdiagnosed because of muscular weakness. Electroencephalographypolymyographic recordings may be needed in these cases 8,9 . The main symptoms in our cases presented here were developmental delay in motor milestones and pes equinovarus deformity. Both of them had occipital cortical dysgenesis, subcortical band heterotopia and bioccipital white matter hyperintensities on brain MRI scans. It is not known why solely occipital cortex dysgenesis occurs in laminin $\alpha 2$ deficiency despite extensive cortex dysgenesis in alpha dystroglycanopathies. Genotype phenotype correlations regarding central nervous system malformations are not clear. The first patient's mutation was also described in a Mexican girl. Although brain magnetic resonance imaging of this patient showed white matter changes, the patient did not have occipital cortical dysgenesis ${ }^{10}$.

In conclusion, infants presenting with muscle weakness, increased serum creatine kinase and occipital dysgenesis with band heterotopia and white matter changes should be examined for laminin $\alpha 2$ deficiency. Knowledge of typical occipital cortex involvement in these cases may lead to mutation analysis of LAMA2 gene and prevent invasive procedures like muscle biopsy.

\section{Acknowledgement}

The authors would like to thank all the patients that contributed to this study. We thank the Cologne Center for Genomics for the next generation sequencing. SC received funding MDA and Deutsche Forschungsgemeinschaft (DFG) to support this work.

\section{REFERENCES}

1. Tome FM, Evangelista T, Leclerc A, et al. Congenital muscular dystrophy with merosin deficiency. C R Acad Sci III 1994; 317: 351-357.

2. Tomoko Yamamoto, Atsuko Hiroi, Makiko Osawa and Noriyuki Shibata. The muscular Dystrophies Associated with Central Nervous System Lesion: A brief Review from a Standpoint of the Localization and Function of Causative Genes. Curr Pediatr Rev; 2014; 10: 282-291.

3. Quijano-Roy S, Sparks S, Rutkowski A. LAMA2-Related Muscular Dystrophy. GeneReviews ${ }^{\circledR}$ [Internet]. Pagon RA, Adam MP, Ardinger HH, et al., editors. Seattle (WA): University of Washington, Seattle; 1993-2016. 2012 Jun 7. https://www.ncbi.nlm.nih.gov/books/ NBK97333/

4. Bönneman $\mathrm{CG}$, Wang $\mathrm{CH}$, Quijano-Roy $\mathrm{S}$, et al. Diagnostic approach to the congenital muscular dystrophies. Neuromuscul Disord 2014; 24: 289-311.

5. Leite CC, Lucato LT, Martin MG, et al. Merosin-deficient congenital muscular dystrophy (CMD): a study of 25 Brazilian patients using MRI. Pediatr Radiol 2005; 35: 572-579.

6. Geranmayeh F, Clement E, Feng LH, et al. Genotypephenotype correlation in a large population of muscular dystrophy patients with LAMA2 mutations. Neuromuscul Dis 2010; 20: 241-250.

7. Tsao CY, Mendell JR, Rusin J, Luquette M. Congenital muscular dystrophy with complete laminin-a-2deficiency, cortical dysplasia, and cerebral white-matter changes in children. J Child Neurol 1998; 13: 253-256.

8. Pini A, Merlini L, Tome FM, Chevallay M, Gobbi G. Merosin-negative congenital muscular dystrophy, occipital epilepsy with periodic spasms and focal cortical dysplasia. Report of three Italian cases in two families. Brain Dev 1996; 18: 316-322.

9. Vigliano P, Dassi P, Di Blasi C, Mora M, Jarre L. LAMA2 stop-codon mutation: merosin-deficient congenital muscular dystrophy with occipital polymicrogyria, epilepsy and psychomotor regression. Eur J Paediatr Neurol 2009; 13: 72-76.

10. Coral-Vazquez RM, Rosas-Vargas H, Meza-Espinosa $M$, et al. Severe congenital muscular dystrophy in a Mexican family with a new nonsense mutation (R2578X) in the laminin alpha-2 gene. J Hum Genet 2003; 48: 91-95. 